\title{
Sarcopenia, Currently a Hot Topic: A Narrative Review
}

\author{
JungHoon Chai ${ }^{1}$, HyunJoo Kang ${ }^{1, *}$ \\ ${ }^{1}$ Department of Sports Medicine, Soonchunhyang University, Korea
}

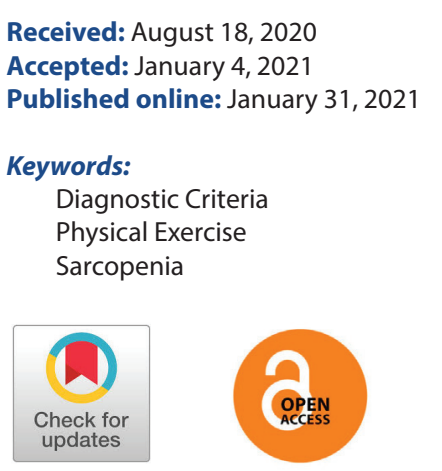

\section{ABSTRACT}

Aging is associated with a progressive decline of skeletal muscle quantity and quality leading to a gradual slowing of movement, a decline in strength and power, and a condition known as sarcopenia. Sarcopenia is a syndrome a risk of adverse outcomes such as physical disability, poor quality of life and death. Agerelated losses in skeletal muscle mass and function have had a significant important in health care issue. In this study, we reviewed the recently introduced criteria for the Eastern and Western diagnosis of sarcopenia. A narrative review of evidence- and non-evidence-based papers was conducted, using a relevant methodological framework. This review is included the following: 1) definitions and diagnostic criteria over time; 2) pathophysiology of muscle loss; 3) management especially protein or amino acid supplementation, medications, and physical exercise.

(c) The Asian Society of Kinesiology and the Korean Academy of Kinesiology

\section{Introduction}

Sarcopenia is a process of aging that means a decrease in muscle strength and function due to a decrease in skeletal muscle [1]. From a pathophysiologic perspective, sarcopenia can be considered an organ failure which can develop chronically or acutely [2]. Clinical studies indicate that sarcopenia is associated with a decreased walking ability, more frequent use of a cane, and difficulty in climbing, lifting, kneeling, or standing up from a chair [3-4]. Sarcopenia is a syndrome a risk of adverse outcomes such as physical disability, poor quality of life and death. In this research, we reviewed the literature to introduce the definitions and diagnostic criteria, pathophysiology and management of sarcopenia with clinical relevance for exercise physiologist. This study is designed to introduce the latest diagnostic methods of sarcopenia and is intended to help the elderly

*Correspondence: HyunJoo Kang, Department of Sports Medicine, Soonchunhyang University, 22 Soonchunhyang-ro, Asan, Chungnam, 31538, Korea; Tel: +82-41-5301280; E-mail: violethjk@hanmail.net to organize exercise programs. The development of criteria for the diagnosis of sarcopenia is complex and must depend upon skeletal muscle mass and functional capacity in geriatric patients, this should draw health care provider. In addition, the effect of exercise being introduced as a preventive measure of sarcopenia is presented.

\section{Definitions}

Sarcopenia was first coined by Irwin Rosenberg in 1989 is now widely accepted to describe the steady and involuntary loss of skeletal muscle mass during aging [8]. Although many challenges exist to make definition of sarcopenia, but until now, there is no consensus. Baumgartner et al. (1998) were the first define sarcopenia as the appendicular skeletal muscle/ height ${ }^{2}\left(\mathrm{ASM} / \mathrm{h}^{2}\right)$ by measuring muscle mass as DXA (dualenergy X-ray absorptiometry) and set the case of less than two standard deviation of young healthy mean as the set-off point of sarcopenia [1]. Second definition of sarcopenia was 
developed by Janssen and colleagues. They measured SMI (\%; total SMI $[\mathrm{kg}] /$ weight $[\mathrm{kg}] \times 100)$ using BIA (bio-electrical impedance analysis) [5]. Newman et al. (2003) indicated that the ASM/height ${ }^{2}$ index primarily identified individuals with low BMI as sarcopenia [6]. Cruz-Jentoft et al. (2010) measured muscle mass as DXA and BIA, cut-off point was same as Baumgartner et al. (1998). Additionally, muscle strength and physical performance were evaluated as grip strength and gait speed or SPPB (short physical performance battery) [3]. Fielding et al. (2011) measured muscle mass using only DXA, and separated men $\left(\leq 7.23 \mathrm{~kg} / \mathrm{m}^{2}\right)$ and women's standards $\left(\leq 5.67 \mathrm{~kg} / \mathrm{m}^{2}\right)$ with appendicular lean mass $/$ height $^{2}$ (ALM/ $\mathrm{h}^{2}$ ). It was defined as physical function was evaluated as gait speed but did not provide a standard for muscle strength [7].

In 2010, the European Working Group on Sarcopenia in Older People (EWGSOP) and the Foundation for the National Institutes of Health (FNIH) established sarcopenia definitions and diagnostic criteria [8]. EWGSOP defined sarcopenia as a gradual decrease in skeletal muscle mass and strength, and reduced muscle mass, strength and physical performance became diagnostic criteria [8]. The International Working Groups on Sarcopenia (IWGS) [7] and the EWGSOP [8] adopted the criterion based on DXA to define sarcopenia by Baumgartner et al (1998) [1].

In 2019, a revised EWSGOP2 has developed new consensus criteria [9]. In EWGSOP2, muscle mass is measured as BIA to calculate ASM $/ \mathrm{h}^{2}$ and body mass index and muscle strength is evaluated by hand grip strength. What makes EWGSOP2 different from EWGSOP is that it categorizes the severity of sarcopenia and sets new cut-off points [10].

Meanwhile, as Asia differs from Europe by ethnicities, cultural and lifestyles, diagnostic criteria of Asian Working Group for Sarcopenia (AWGS) different from EWGSOP are introduced [11]. In AWGS, muscle mass was measured as DXA and BIA and the result calculated as skeletal muscle mass $/ h^{2}$ was used as an evaluation variable, and muscle strength was evaluated as hand grip strength in the same way as EWGSOP. The AWGS was also revised in 2019, and criteria for questionnaire and calf circumference were introduced to help people easily recognize the risk of sarcopenia, and in addition, diagnostic methods such as 6-minute walking, SPPB, and chair stand test were defined [12].

Numerous epidemiological studies using different methods of measurement and cut-off points have attempted to establish the prevalence of sarcopenia. In general, 5-13\% of population aged $60-70$ years and $11-50 \%$ of population in their 80 s have been assumed to have sarcopenia. Thus, 3.6 million persons in the USA were estimated to be with sarcopenia [13-16].

\section{Diagnostic Criteria}

Various methods were introduced to assess sarcopenia. Measuring skeletal muscle mass is a very direct and useful way to assess sarcopenia. BIA and anthropometry are also very useful method and popular one to assess sarcopenia, because of the noninvasiveness and absence of radiation hazard. These electrical equipment measures electrical resistance, skinfold thickness and circumferences. These factors make it possible to predict muscle mass. However, the limitation is its vulnerability for hydration and systemic error while collecting data.

Recently, DXA, magnetic resonance imaging (MRI), and computed tomography (CT) were suggested to assess whole body skeletal muscle. However, these contain limitation of radiation exposure and cost issue [16].

Baumgartner et al. (1998) developed the operational definition of sarcopenia first [1]. The approach was defined as ASM (sum of the masses of arm and leg lean soft tissue from DXA) divided by height squared (also referred to as stature, $\mathrm{ASM} / \mathrm{S}^{2}$ ). They used statistics to get cutoff values. The two standard deviation below sex-specific mean of the distributions in a reference sample of young and middle-aged adults from the Rosetta Study was the criteria [14]. Other studies reported sarcopenia prevalence in different age, racial and gender characteristics, using various method [17-18]. For example, Melton et al. (2000) proposed different sarcopenia index defined as total lean body mass/stature ${ }^{2}$ [18], Janssen et al. (2004) used BIA to calculate the index defining total skeletal muscle/stature ${ }^{2}$ in US population [5].

In EWGSOP2, ASM/ $\mathrm{h}^{2}$ and body mass index (ASM/BMI) calculated as BIA, and cut-off was set to $<7.0 \mathrm{~kg} / \mathrm{m}^{2}$ for men and $<5.5 \mathrm{~kg} / \mathrm{m}^{2}$ for women. In addition, cut-off point was set 
to ASM/BMI ratio less than $<0.789$ for men and $<0.512$ for women. On the other hand, muscle strength was evaluated as grip strength, and cut-off point was set to $<27 \mathrm{~kg}$ for men and $<16 \mathrm{~kg}$ for women [11]. Gait speed is used to classify the severity of sarcopenia [10].

AWGS evaluates muscle mass as DXA and BIA. Cut-off point of DXA is set to less than $<7.0 \mathrm{~kg} / \mathrm{m}^{2}$ for men and $<5.4 \mathrm{~kg} /$ $\mathrm{m}^{2}$ for women. BIA was calculated as skeletal muscle mass/ $\mathrm{h}^{2}$ and set to less than $<7.0 \mathrm{~kg} / \mathrm{m}^{2}$ for men and $<5.7 \mathrm{~kg} / \mathrm{m}^{2}$ for women. The cut-off point of grip strength is $<26 \mathrm{~kg}$ for men and $<18 \mathrm{~kg}$ for women [12]. The criteria for calf circumference were $<34 \mathrm{~cm}$ for men and $<33 \mathrm{~cm}$ for women, and the score criteria for questionnaires were also presented (SARC-F $\geq 4$ or SARC-CalF $\geq 11$ ). Finally, the modified AWGS (2019) should not only diagnose sarcopenia, but also monitor its potential [12].

Over the years, no relevant consensus has been reached to find the definition and diagnostic criteria of sarcopenia until now. However, the fact that the best measures are based on muscle strength rather than muscle mass particularly in the context of cardiovascular disease has been confirmed [19].

\section{Pathophysiology of Skeletal Muscle Loss}

Sarcopenia can cause various clinical problems, such as loss of strength, mobility disorders, disability, and poor quality of life [4, 10, 20-22]. Various factors including vitamin D deficiency, pro-inflammatory cytokines, decreasing growth hormones etc., can cause sarcopenia [7].

The role of apoptosis in post-mitotic tissues such as skeletal muscle is not clearly understood [23]. From the cellular level, studies proved that different conditions leading to muscle wasting involved different cell signaling pathways that might lead to programmed cell death (apoptosis), increased protein breakdown, or even decreased activation of the satellite cells responsible for muscle regeneration (Figure 1).

Mauro (1961) observed a group of mononucleated cells at the periphery of adult skeletal muscle myofibers by electron microscopy. These were named satellite cells due to their sublaminar location and intimate association with the plasma membrane of myofibers. The direct juxtaposition of

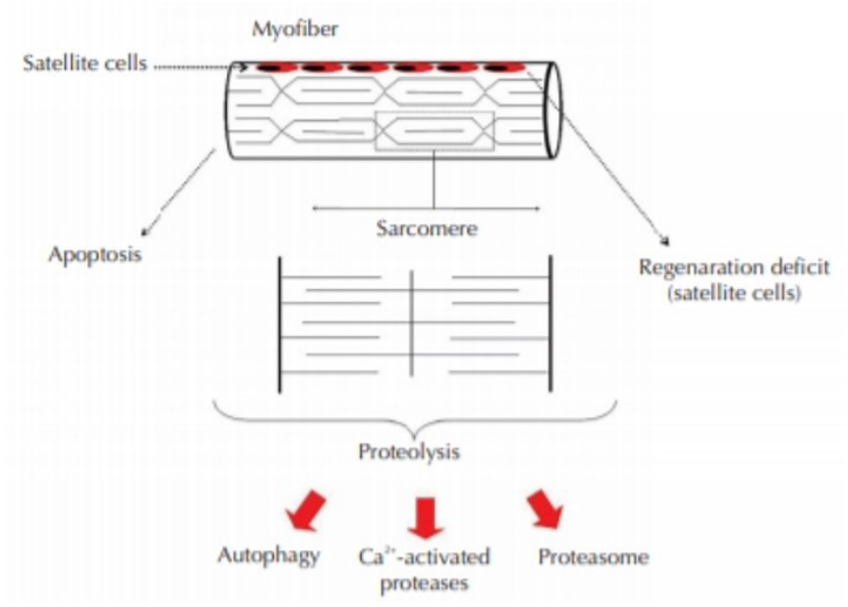

Figure 1. Apoptosis of Skeletal muscle. (Teixeira et al., 2012) [23]

satellite cells and myofibers immediately raised a hypothesis that these cells may be involved in skeletal muscle growth and regeneration [24]. Homeostatic and regenerative replacement of skeletal muscle fibers requires the activity of satellite cells. Satellite cell function is controlled by both intrinsic and extrinsic regulatory cues, and pathological deregulation of satellite cell function has been proposed to play an important role in age-dependent deterioration of muscle function and in muscle dystrophic disease. However, satellite cell transplantation to enhance muscle repair or restoration has little data in the literature.

It is very clear that sarcopenia is positively associated with various physical and physiological problem, but the problem is that it is very hard to know which one comes first. However, aging is the prime factor for this condition. Recent studies described the detailed mechanism of the sarcopenia in the elderly and found that one of the most prominent causes of sarcopenia is inactivity [25] (Figure 2). Exercise stimulates the release of muscle growth factors to activate satellite cells and protein synthesis. The regenerated muscle can delay the aging process. In other word, aging process accompanied by sarcopenia can be accelerated by immobilization. Sarcopenia can be aggravated by itself, because of the decreased physical function can also cause sarcopenia.

\section{Management}

In recent years, there has been an increased interest in the 


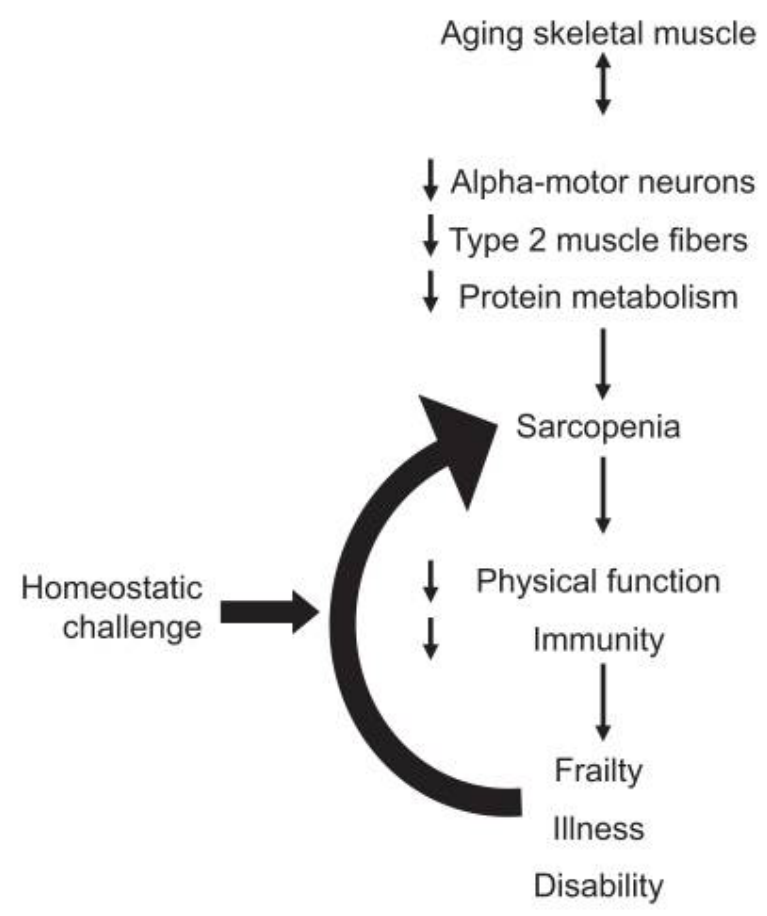

Figure 2. Proposed downward spiral of muscle loss. (Waters DL et al., 2010) [25]

treatment and management of sarcopenia. The management of sarcopenia should focus on improving muscle mass and physical function. Researchers have confirmed that nutritional support, medication, and physical exercise play important roles in the treatment of sarcopenia. Consuming the proper amounts of dietary protein and physical exercise may lead to a reduction in muscle loss and physical function.

\section{Protein and Amino Acid Supplementation}

Nutrition is the first key element as for treatment and prophylaxis to solve sarcopenia. Morley suggested daily intake of $1.2 \sim 1.5 \mathrm{~g} / \mathrm{kg}$ of protein to prevent sarcopenia [16]. Recent randomized controlled trial indicates that it is important to ingest a sufficient amount of high-quality protein (25-30g) with each meal rather than one large bolus [26]. Several studies revealed that the nonessential amino acids are also required in a nutritional supplement to stimulate muscle protein anabolism [27], because the essential amino acid leucine increases protein anabolism and decreases protein breakdown [28]. Researches are needed to be determine if the quality of the dietary protein and amino acid supplementation enhance skeletal muscle. In addition, the mechanism of action of the effect of protein and amino acid support with physical exercise on skeletal muscle and muscle strength.

\section{Medication}

Medication is another important regime to manage sarcopenia. Various hormones such as testosterone, estrogen, growth hormone, and creatine, angiotensin II converting enzyme inhibitors (ACEIs), anti-myostatin, specific androgen receptor modular (SARMs), and others were suggested for sarcopenia. Among them, creatine, ACEIs, myostatin are most commonly accepted medical therapeutic agents. Creatine from meat is potential ergogenic aid due to its buffering action against proton accumulation [29]. ACEIs works for skeletal muscle by suppressing the angiotensin-aldosterone system, reducing the pre- and afterload on the heart improving myocardial contractility Although the mechanisms of ACEIs are still not clear, it is obvious that ACEIs can improve cardiac output and improve blood flow to muscle, reduce inflammatory cytokines, etc [30-31]. Myostatin play important role as negative regulator of skeletal muscle myogenesis [3234]. There are numerous potential targets for increasing muscle mass and strength, and the development of new medication for sarcopenia represents a possibly exciting clinical field. A better understanding of the molecular changes behind sarcopenia will help researchers develop better therapies to improve the skeletal muscle of elderly persons.

\section{Physical Exercise}

Successful aging is highly related to physical fitness levels [35]. Exercise is also very important factors to prevent sarcopenia [36]. Resistance exercise increases muscle mass, muscular strength, and physical function, whereas aerobic exercise improves cardiopulmonary capacity. Recent evidence on resistance training supports earlier research that it may be the most effective strategy to combat sarcopenia though muscle hypertrophy and increased muscular strength and power [37]. In addition, research has focused on the impact of resistance training on muscular strength rather than power, which is the product of force and speed [38]. 
Borde et al. (2015) reported that when exercising with an intensity of $70-79 \%$ of $1 \mathrm{RM}$, it is the most effective for improving muscle strength [39]. Jung et al. (2019) also reported that high-intensity circuit training improves muscle mass and strength, body composition, balance and pulmonary function in sarcopenia patients [40]. Therefore, it is necessary to continuously explore effective exercise components in addition to recognizing and applying the importance of resistance exercise to the elderly with sarcopenia [41]. The effectiveness and outcomes of this resistance exercise depends on intensity, sets by repetitions, frequency, resistance type, and periods of training. Recent evidence from meta-analysis has revealed that resistance exercise may be actual in enhancing not only appendicular muscle mass, but also knee extension strength and timed up and go in elderly diagnosed with sarcopenia [41].

The endurance exercise increases the maximum aerobic capacity, so when constructing an exercise program for frail older people, and appropriate balance of endurance and strength exercises is necessary [42-43]. Falls in older adults under the influence of sarcopenia also emphasize the importance of exercise, and in addition to strength and power, a balance exercise based on ability is recommended [44-46]. However, since an exercise program suitable for the elderly should be considered to safety, a group exercise that can be supervised and encouraged is recommended [47]. In addition to this, steady frequency, sufficient warm-up, short-term or long-term goals, and nutrition should be considered essential components [48].

\section{Conclusions}

Sarcopenia is an emerging problem considering the rapid growth of the elderly population these days. To reduce the socioeconomic, it is critical to focus on sarcopenia, which is aging related condition resulting in skeletal muscle loss. Pathophysiology illustrates that sarcopenia is strongly associated with gerontology and its accompanying problems. A multidisciplinary approach including nutrition, exercise and medical management are essential to approach effectively treat this emerging condition. In addition, exercise intervention is an economical and cost-effective method of prevention, so exercise specialists need to understand the characteristics of sarcopenia and construct exercise programs. In a future study, it is necessary to find a more effective exercise program by applying various programs introduced as exercise intervention for the elderly and comparing them with the diagnostic criteria of sarcopenia.

\section{Acknowledgments}

This work was supported by the Ministry of Trade, Industry and Energy (MOTIE, Korea) (Project Number: P0004713] and the Soonchunhyang University research fund.

\section{Conflicts of Interest}

The authors declare no conflict of interest.

\section{References}

1. Baumgartner RN, Koehler KM, Gallagher D, et al. Epidemiology of sarcopenia among the elderly in New Mexico. Am J Epidemiol. 1998; 147(8):755-763.

2. Marzetti E, Calvani R, Tosato M, et al. Sarcopenia: an overview. Aging Clin Exp Res. 2017; 29(1):11-17.

3. Cruz-Jentoft AJ, Landi F, Topinková E, Michel JP. Understanding sarcopenia as a geriatric syndrome. Curr Opin Clin Nutr Metab Care. 2010; 13(1):1-7.

4. Shaw SC, Dennison EM, Cooper C. Epidemiology of Sarcopenia: Determinants Throughout the Lifecourse. Calcif Tissue Int. 2017; 101(3):229-247.

5. Janssen I, Heymsfield SB, Ross R. Low relative skeletal muscle mass (sarcopenia) in older persons is associated with functional impairment and physical disability. J Am Geriatr Soc. 2002; 50(5):889-896.

6. Newman AB, Kupelian V, Visser M, et al. Sarcopenia: alternative definitions and associations with lower extremity function. J Am Geriatr Soc. 2003; 51(11):1602-1609.

7. Fielding RA, Vellas B, Evans WJ, et al. Sarcopenia: an undiagnosed condition in older adults. Current consensus definition: prevalence, etiology, and 
consequences. International working group on sarcopenia. J Am Med Dir Assoc. 2011; 12(4):249-256.

8. Cruz-Jentoft AJ, Baeyens JP, Bauer JM, et al. Sarcopenia: European consensus on definition and diagnosis: Report of the European Working Group on Sarcopenia in Older People. Age Ageing. 2010; 39(4):412-423.

9. Cruz-Jentoft AJ, Bahat G, Bauer J, et al. Sarcopenia: revised European consensus on definition and diagnosis. Age Ageing. 2019; 48(1):16-31.

10. Van Ancum JM, Alcazar J, Meskers CGM, Nielsen BR, Suetta C, Maier AB. Impact of using the updated EWGSOP2 definition in diagnosing sarcopenia: A clinical perspective [published online ahead of print, 2020 May 23]. Arch Gerontol Geriatr. 2020; 90:104-125.

11. Chen LK, Lee WJ, Peng LN, et al. Recent Advances in Sarcopenia Research in Asia: 2016 Update from the Asian Working Group for Sarcopenia. J Am Med Dir Assoc. 2016; 17(8):767.e1-7.

12. Chen LK, Woo J, Assantachai P, et al. Asian Working Group for Sarcopenia: 2019 Consensus Update on Sarcopenia Diagnosis and Treatment. J Am Med Dir Assoc. 2020; 21(3):300-307.

13. Tankó LB, Movsesyan L, Mouritzen U, Christiansen C, Svendsen OL. Appendicular lean tissue mass and the prevalence of sarcopenia among healthy women. Metabolism. 2002; 51(1):69-74.

14. Baumgartner RN. Body composition in healthy aging. Ann N Y Acad Sci. 2000; 904:437-448.

15. Gillette-Guyonnet S, Nourhashemi F, Andrieu S, et al. Body composition in French women $75+$ years of age: the EPIDOS study. Mech Ageing Dev. 2003; 124(3):311316.

16. Morley JE. Sarcopenia: diagnosis and treatment. J Nutr Health Aging. 2008; 12(7):452-456.

17. Mott JW, Wang J, Thornton JC, Allison DB, Heymsfield SB, Pierson RN Jr. Relation between body fat and age in 4 ethnic groups. Am J Clin Nutr. 1999; 69(5):1007-1013.

18. Melton LJ 3rd, Khosla S, Crowson CS, O’Connor MK, O’Fallon WM, Riggs BL. Epidemiology of sarcopenia. J Am Geriatr Soc. 2000; 48(6):625-630.

19. Stephen WC, Janssen I. Sarcopenic-obesity and cardiovascular disease risk in the elderly. J Nutr Health Aging. 2009; 13(5):460-466.

20. Lang T, Streeper T, Cawthon P, Baldwin K, Taaffe DR, Harris TB. Sarcopenia: etiology, clinical consequences, intervention, and assessment. Osteoporos Int. 2010; 21(4):543-559.

21. Lloyd BD, Williamson DA, Singh NA, et al. Recurrent and injurious falls in the year following hip fracture: a prospective study of incidence and risk factors from the Sarcopenia and Hip Fracture study. J Gerontol A Biol Sci Med Sci. 2009; 64(5):599-609.

22. Marcell TJ. Sarcopenia: causes, consequences, and preventions. J Gerontol A Biol Sci Med Sci. 2003; 58(10):M911-M916.

23. Teixeira Vde O, Filippin LI, Xavier RM. Mechanisms of muscle wasting in sarcopenia. Rev Bras Reumatol. 2012; 52(2):252-259.

24. Mauro A. Satellite cell of skeletal muscle fibers. J Biophys Biochem Cytol. 1961; 9(2):493-495.

25. Waters DL, Baumgartner RN, Garry PJ, Vellas B. Advantages of dietary, exercise-related, and therapeutic interventions to prevent and treat sarcopenia in adult patients: an update. Clin Interv Aging. 2010; 5:259-270.

26. Symons TB, Sheffield-Moore M, Wolfe RR, PaddonJones D. A moderate serving of high-quality protein maximally stimulates skeletal muscle protein synthesis in young and elderly subjects. J Am Diet Assoc. 2009; 109(9):1582-1586.

27. Volpi E, Kobayashi H, Sheffield-Moore M, Mittendorfer B, Wolfe RR. Essential amino acids are primarily responsible for the amino acid stimulation of muscle protein anabolism in healthy elderly adults. Am J Clin Nutr. 2003; 78(2):250-258.

28. Paddon-Jones D, Rasmussen BB. Dietary protein recommendations and the prevention of sarcopenia. Curr Opin Clin Nutr Metab Care. 2009; 12(1):86-90.

29. Tarnopolsky MA, Safdar A. The potential benefits of creatine and conjugated linoleic acid as adjuncts to resistance training in older adults. Appl Physiol Nutr Metab. 2008; 33(1):213-227. 
30. Onder G, Penninx BW, Balkrishnan R, et al. Relation between use of angiotensin-converting enzyme inhibitors and muscle strength and physical function in older women: an observational study. Lancet. 2002; 359(9310):926-930.

31. Strazzullo P, Iacone R, Iacoviello L, et al. Genetic variation in the renin-angiotensin system and abdominal adiposity in men: the Olivetti Prospective Heart Study. Ann Intern Med. 2003; 138(1):17-23.

32. Langley B, Thomas M, Bishop A, Sharma M, Gilmour S, Kambadur R. Myostatin inhibits myoblast differentiation by down-regulating MyoD expression. J Biol Chem. 2002; 277(51):49831-49840.

33. Thomas M, Langley B, Berry C, et al. Myostatin, a negative regulator of muscle growth, functions by inhibiting myoblast proliferation. J Biol Chem. 2000; 275(51):40235-40243.

34. McCroskery S, Thomas M, Maxwell L, Sharma M, Kambadur R. Myostatin negatively regulates satellite cell activation and self-renewal. J Cell Biol. 2003; 162(6):1135-1147.

35. Lin PS, Hsieh CC, Cheng HS, Tseng TJ, Su SC. Association between Physical Fitness and Successful Aging in Taiwanese Older Adults. PLoS One. 2016; 11(3):e0150389.

36. Cartee GD, Hepple RT, Bamman MM, Zierath JR. Exercise Promotes Healthy Aging of Skeletal Muscle. Cell Metab. 2016; 23(6):1034-1047.

37. de Vos NJ, Singh NA, Ross DA, Stavrinos TM, Orr R, Fiatarone Singh MA. Effect of power-training intensity on the contribution of force and velocity to peak power in older adults. J Aging Phys Act. 2008; 16(4):393-407.

38. Perry MC, Carville SF, Smith IC, Rutherford OM, Newham DJ. Strength, power output and symmetry of leg muscles: effect of age and history of falling. Eur J Appl Physiol. 2007; 100(5):553-561.
39. Borde R, Hortobágyi T, Granacher U. Dose-Response Relationships of Resistance Training in Healthy Old Adults: A Systematic Review and Meta-Analysis. Sports Med. 2015; 45(12):1693-1720.

40. Jung WS, Kim YY, Park HY. Circuit Training Improvements in Korean Women with Sarcopenia. Percept Mot Skills. 2019; 126(5):828-842.

41. Kim KM, Kang HJ. Effects of Resistance Exercise on Muscle Mass, Strength, and Physical Performances in Elderly with Diagnosed Sarcopenia: A Systematic Review and Meta-Analysis. Exerc Sci. 2020; 29(2):109-120.

42. Landi F, Marzetti E, Martone AM, Bernabei R, Onder G. Exercise as a remedy for sarcopenia. Curr Opin Clin Nutr Metab Care. 2014; 17(1):25-31.

43. Cooper C, Dere W, Evans W, et al. Frailty and sarcopenia: definitions and outcome parameters. Osteoporos Int. 2012; 23(7):1839-1848.

44. Gschwind YJ, Kressig RW, Lacroix A, Muehlbauer T, Pfenninger B, Granacher U. A best practice fall prevention exercise program to improve balance, strength / power, and psychosocial health in older adults: study protocol for a randomized controlled trial. BMC Geriatr. 2013; 13:105.

45. Thomas E, Battaglia G, Patti A, et al. Physical activity programs for balance and fall prevention in elderly: A systematic review. Medicine (Baltimore). 2019; 98(27):e16218.

46. Donath L, van Dieën J, Faude O. Exercise-Based Fall Prevention in the Elderly: What About Agility?. Sports Med. 2016; 46(2):143-149.

47. Zão A, Camelo AP. Exercise for Sarcopenia in the Elderly: What Kind, Which Role? Research \& Investigations in Sports Medicine. 2018; 2(1):9-11.

48. Phu S, Boersma D, Duque G. Exercise and Sarcopenia. J Clin Densitom. 2015; 18(4):488-492. 